\title{
PCR Genetic Expression of Interleukin 37 in the Eutopic and Ectopic Endometrium of Women with Endometriosis
}

Ahmed Mohamed Abbas ${ }^{1}$, Sayeda Ibrahim EL-Desouky², Ihab Fouad Serag Eldin Allam ${ }^{1}$, Deena A Mohamed ${ }^{3}$, Amgad Alsaid Abou-Gamrah ${ }^{1}$

Departments of ${ }^{1}$ Obstetrics \& Gynecology and Clinical \& Chemical Pathology ${ }^{3}$, Faculty of Medicine, Ain Shams University

${ }^{2}$ Banha Teaching Hospital

*Corresponding author: Sayeda Ibrahim EL-Desouky, Mobile: (+20)01005414292, E-mail: noonnoun@ hotmail.com

\begin{abstract}
Background: In endometriosis, there is a chronic immune and inflammatory reaction.

Objective: To study the PCR genetic expression of interleukin 37 in the eutopic and ectopic endometrium of women with endometriosis in comparison with controls.

Patient and Methods: Forty-six patients were included in the study. Twenty-three women diagnosed as endometriosis and twenty-three women diagnosed as free of endometriosis were subjected to laparoscopy for any other gynecological cause. Interleukin 37 gene expression was studied in ectopic and eutopic endometrium using polymerase chain reaction.

Results: Ectopic endometrium expressed high levels of mRNA compared to eutopic endometrium in endometriosis patients. Eutopic endometrium of endometriosis patients expressed high levels of mRNA compared to eutopic endometrium of controls.

Conclusion: The finding of high mRNA produced in endometrial tissue suggests that interleukin 37 may be important in the chronic inflammation occurring during endometriosis.
\end{abstract}

Keywords: Endometriosis, PCR Genetic Expression, Interleukin 37

\section{INTRODUCTION}

Endometriosis is defined as the presence of endometrial-like tissue outside the uterine cavity. It is a common, chronic, progressive and inflammatory disease, associated with local and systemic altered immune system. It is an estrogen-dependent pelvic inflammatory disease affecting about $10-15 \%$ of women in reproductive age ${ }^{(\mathbf{1})}$. More than 180 million women worldwide are affected by the disease. The definition of endometriosis is histological so it requires the identification of the presence of endometrial gland and stroma-like tissue outside (ectopic) the uterus. These ectopic lesions are commonly located on the pelvic organs and peritoneum. Most common affected sites are the ovaries, fallopian tubes and tissues around the uterus ${ }^{(2)}$.

Further insights into the pathogenesis of endometriosis could be valuable, possibly leading to new therapeutic approaches. Mechanisms underlying the chronicity of this disease are not understood, but are of major importance in its pathogenesis. There has been much speculation about the role of immune mediators in the pathogenesis of endometriosis, especially cytokines. This was based on the presence of different cytokines in both endometriosis tissue, peritoneal fluid and high levels of cytokines in serum of endometriosis patients. Cytokines have proinflammatory actions on the surrounding tissue, stimulating inflammation and angiogenesis ${ }^{(3)}$.
Interleukin- 37 (IL-37) (previously called IL1F7) is a recently discovered member of the IL-1 family. It is located both in the cytoplasm and in the nucleus. The key role of IL-37 is that it inhibits both innate and adaptive immunity by decreasing expression of pro-inflammatory cytokines (4). Interleukin-37, located within the IL-1 gene cluster on $2 \mathrm{q} 12$, has recently been identified as a regulatory cytokine that dampens the immune response in humans ${ }^{(5)}$. A previous study reported that the immunostaining score of IL-37 was significantly higher in the eutopic endometrium $(4.79 \pm 1.91)$ and ectopic endometrium $(7.71 \pm 1.78)$ of women with ovarian endometriosis compared to that of controls without endometriosis $(3.27 \pm 1.62)^{(6)}$.

The present work aimed to study the PCR genetic expression of interleukin 37 in the eutopic and ectopic endometrium of women with endometriosis in comparison with controls.

\section{PATIENTS AND METHODS}

This case-control study was conducted in Ain Shams University Maternity Hospital in Laparoscopy Department in the period from October 2016 to October 2018. Forty-six patients were included in the study where twenty-three women were diagnosed as endometriosis and twenty-three women were free of endometriosis and were subjected to laparoscopy for any other gynecological cause. 
All women in the study were subjected to the following: 1) Complete history taking including name, age, occupation, address, habits, complaint, present history of any medical problem, medication taken in treatment of infertility, past history of previous operation, heart disease, D.M., renal disease, liver disease and family history of medical diseases as D.M. or hypertension. Obstetric history (if it was present): in the form of: antenatal care, intranatal care and mode of delivery. Menstrual history: History of dysmenorrhea and its relation to the menstrual cycle, chronic pelvic pain and dyspareunia.

2) General examination: Vital signs, arterial blood pressure, pulse, temperature, respiratory rate and heart rate.

3) Abdominal examination: Inspection of scar of previous operation.

4) Investigations: Complete blood cell count, liver function tests and renal function tests.

\section{Ethical approval}

An approval of the study was obtained from Ain Shams University academic and ethical committee.

All women included in this study were informed about the aim of this work and a signed written consent was taken from every woman participated in the study.

The matched eutopic endometrium collected from the patient with endometriosis by curettage and the matched ectopic endometrium collected from the same patient from the peritoneal cavity by laparoscopy at the same time were used for diagnosis. The diagnosis of ovarian endometriosis must be confirmed by histopathological examination after the surgery. In addition, endometriosis was scored and staged according to the revised classification system of the
American Fertility Society (rAFS). Tissues collected immediately after completion of the surgery were frozed and preserved at $-80{ }^{\circ} \mathrm{C}$ for polymerase chain reaction.

Total cellular RNA from tissue was isolated by using RNeasy micro kit extraction columns (Qiagen, Chatsworth, CA, USA). RNA was reversetranscribed using Superscript II reverse transcriptase (Invitrogen, Life Technologies Products, USA).

Quantification of mRNA levels was performed using QuantitTect SYBR Green PCR master mix (Qiagen, Chatsworth, CA, USA). Primers were as follows: IL-37, forward: 5' CTCCTGGGGGTCTCTAAAGG-3' and reverse $5^{\prime}$ TACAATTGCAGGAGGTGCAG-3'(reverse); $\beta$-actin, forward 5'-CCTGACTGACTACCTCATGAAG-3' and reverse: 5'-GACGTAGCACAGCTT CTCCTTA3'. Relative mRNA levels of target gene were calculated by the $2^{-\Delta \Delta c t}$ method.

\section{Statistical analysis}

Recorded data were analyzed using the statistical package for social sciences, version 20.0 (SPSS Inc., Chicago, Illinois, USA). Quantitative data were expressed as mean \pm standard deviation (SD). Qualitative data were expressed as frequency and percentage. Independentsamples t-test of significance was used when comparing between two means. Chi-square $\left(\mathrm{x}^{2}\right)$ test of significance was used in order to compare proportions between two qualitative parameters.

The confidence interval was set to $95 \%$ and the accepted margin of error was set to 5\%. The pvalue was considered significant as the following: $\mathrm{P}$ value $\leq 0.05$ was considered significant, $\mathrm{P}$-value < 0.001 was considered as highly significant and P-value $>0.05$ was considered insignificant.

\section{RESULTS}

No statistically significant differences were found between women of both groups regarding age or parity (Table1).

Table (1): Comparison between study groups regarding basic demographic characteristics

\begin{tabular}{|l|c|c|c|}
\hline & Endometriosis Group & Control Group & P \\
\hline Age (Yrs) & $18.0-42.0$ & $19.0-38.0$ & 0.87 \\
Range & $26.95 \pm 5.98$ & $26.70 \pm 4.73$ & \\
Mean \pm SD & $0-2$ & $0-2$ & 0.46 \\
\hline Parity & $0(0-1)$ & $0(0-1)$ & \\
Range & & & \\
Median (IQR) & & & \\
\hline
\end{tabular}

In addition, no statistically significant differences were found in the indications for laparoscopy in the two groups. The performed laparoscopic procedures, as well as the rAFS scoring and staging for endometriosis are listed in table (2). It should be noted that the considerable overlap in the performed procedures in the participants, e.g. in some women, both ovarian cystectomy and tubal disconnection were done in the same setting. 
Table (2): Comparison between study groups regarding laparoscopic characteristics

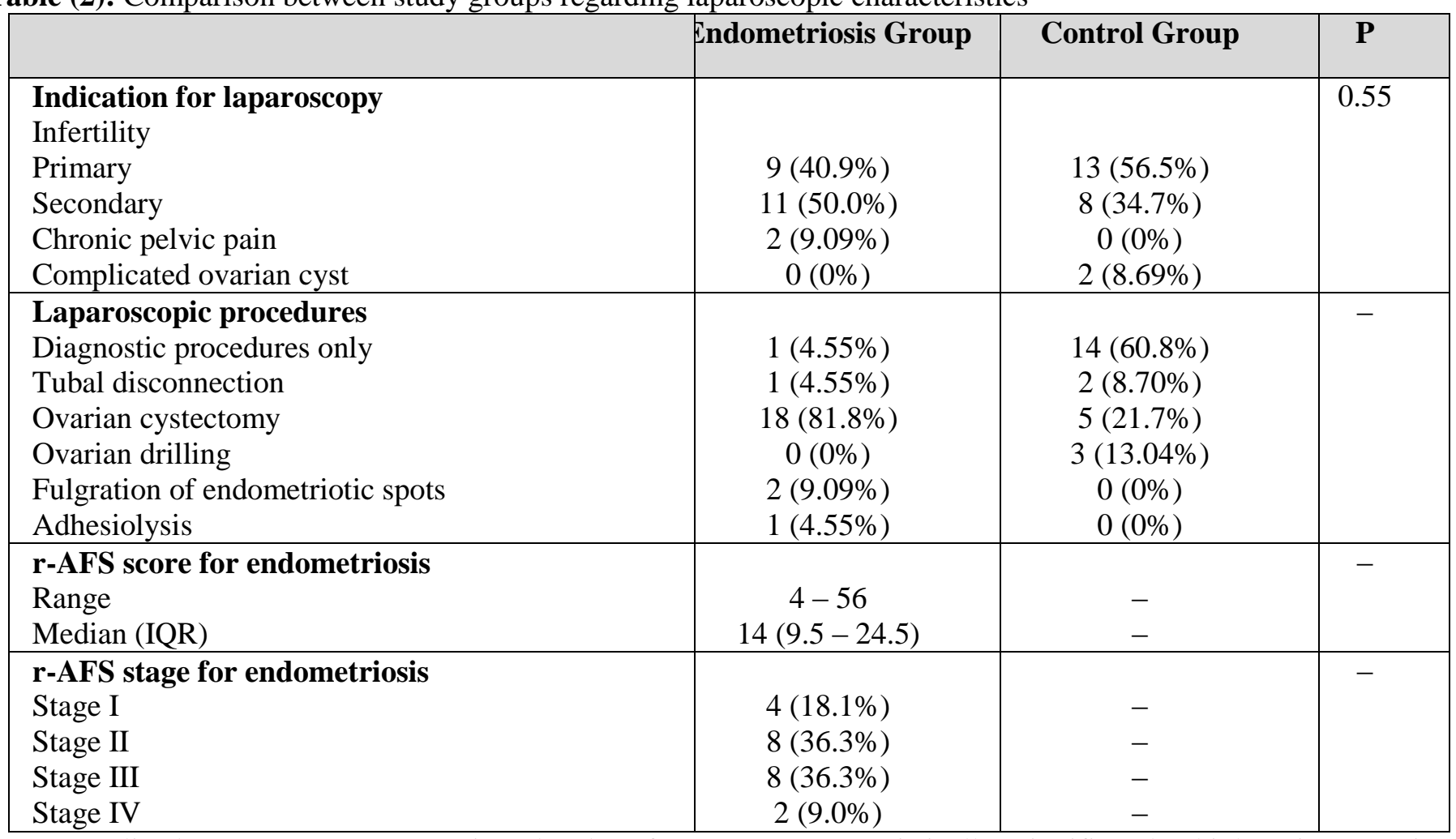

Normalized target gene expression levels of IL-37 were statistically significantly higher in eutopic endometriosis of the endometriosis group compared to the eutopic endometriosis of control group (Table 3).

Table (3): Comparison between normalized target gene expression fold changes (2- $\Delta \Delta \mathrm{CT})$ in eutopic endometrial tissues of the two study groups

\begin{tabular}{|l|c|c|c|}
\hline & $\begin{array}{c}\text { Endometriosis } \\
\text { Group }\end{array}$ & $\begin{array}{c}\text { Control } \\
\text { Group }\end{array}$ & P \\
\hline $\begin{array}{l}\text { Normalized target gene expression } \\
\text { level }(\mathbf{2}-\boldsymbol{\Delta} \mathbf{\Delta C T}) \text { in eutopic } \\
\text { endometrium }\end{array}$ & & & $<\mathbf{0 . 0 0 1}$ \\
Range & $8.50-45.60$ & $0.33-11.38$ & \\
Mean \pm SD & $20.78 \pm 10.32$ & $5.40 \pm 2.94$ & \\
$95 \%$ CI & $16.21-25.36$ & $4.12-6.67$ & \\
\hline
\end{tabular}

Paired comparison of normalized target gene expression levels in ectopic endometrium and eutopic endometrium revealed statistically significantly higher levels in the ectopic endometrium compared to the eutopic endometrial tissue in the endometriosis group (Table 4).

Table (4): Paired comparison between normalized target gene expression fold changes $(2-\Delta \Delta \mathrm{CT})$ in ectopic endometrial tissues and eutopic endometrial tissues in patients groups

\begin{tabular}{|l|c|c|c|}
\hline & $\begin{array}{c}\text { Normalized target gene } \\
\text { expression level in ectopic } \\
\text { endometrium }\end{array}$ & $\begin{array}{c}\text { Normalized target gene } \\
\text { expression level in eutopic } \\
\text { endometrium }\end{array}$ & P \\
\hline Endometriosis group & $9.32-61.78$ & $8.50-45.60$ & $<\mathbf{0 0 0 1}$ \\
Range & $32.17 \pm 14.34$ & $20.78 \pm 10.32$ & \\
Mean \pm SD & $25.81-38.52$ & $16.21-25.36$ & \\
\hline $95 \%$ CI &
\end{tabular}

No statistically significant correlation was found between normalized target gene expression levels in women with endometriosis of either eutopic endometrium or ectopic endometrium and age or parity. On the other hand, normalized target gene expression in both eutopic endometrium and ectopic endometrium was positively correlated with rAFS score and stage (Table 5 and Figure 1). 
Table (5): Correlation between normalized target gene expression fold changes in eutopic endometrium and ectopic endometrium in women with endometriosis and age, parity and rAFS endometriosis score/stage

\begin{tabular}{|c|c|c|c|c|}
\hline & \multicolumn{2}{|c|}{$\begin{array}{l}\text { ormalized target gene expression } \\
\text { level in eutopic endometrium }\end{array}$} & \multicolumn{2}{|c|}{$\begin{array}{l}\text { Normalized target gene expression } \\
\text { level in ectopic endometrium }\end{array}$} \\
\hline & r $(95 \% \mathrm{CI})$ & $\mathbf{P}$ & $\mathbf{r}(95 \% \mathrm{CI})$ & $\mathbf{p}$ \\
\hline Age & $\begin{array}{c}-0.12 \\
(-0.54-0.34)\end{array}$ & 0.60 & $\begin{array}{c}-0.12 \\
(-0.54-0.35)\end{array}$ & 0.61 \\
\hline Parity & $\begin{array}{c}0.007 \\
(-0.42-0.43)\end{array}$ & 0.97 & $\begin{array}{c}-0.26 \\
(-0.62-0.18)\end{array}$ & 0.23 \\
\hline rAFS endometriosis score & $\begin{array}{c}0.70 \\
(0.39-0.86)\end{array}$ & $<0.001$ & $\begin{array}{c}0.74 \\
(0.47-0.88)\end{array}$ & $<0.001$ \\
\hline rAFS endometriosis stage & $\begin{array}{c}0.72 \\
(0.43-0.87)\end{array}$ & $<0.001$ & $\begin{array}{c}0.83 \\
(0.63-0.92)\end{array}$ & $<0.001$ \\
\hline
\end{tabular}

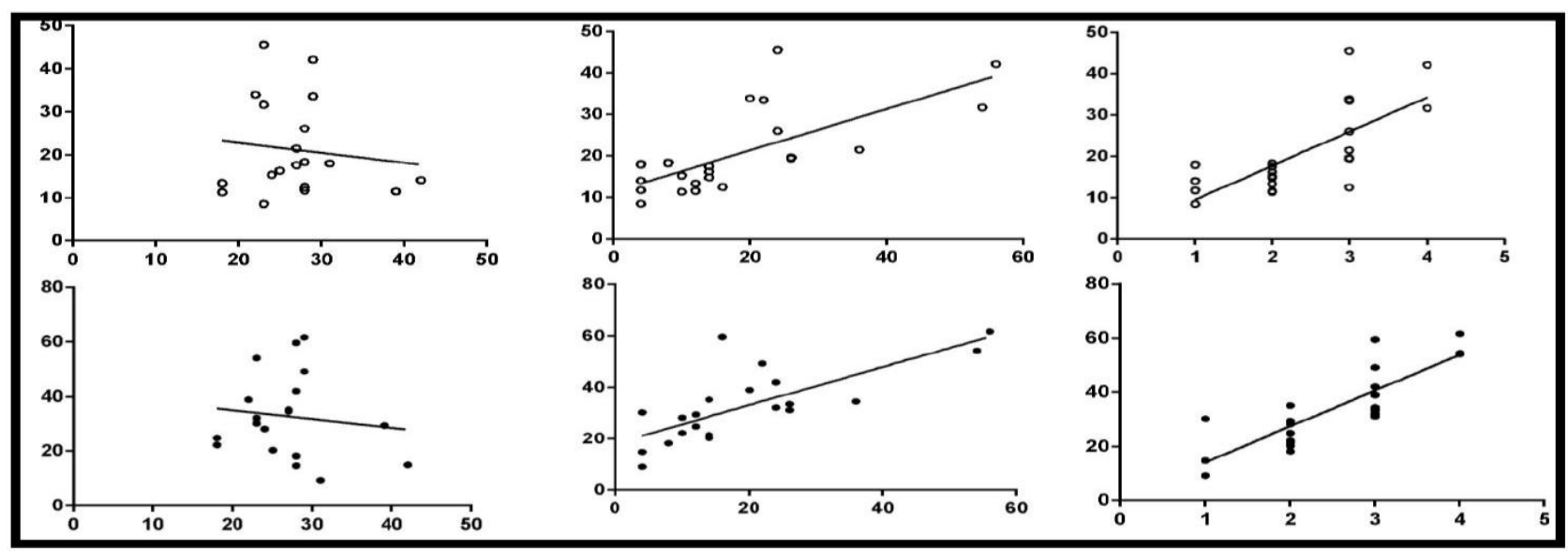

Fig. (1): Correlation between normalized target gene expression (y-axis) in eutopic endometrium (upper) and ectopic endometrium (lower) and age (left), rAFS score (middle) and rAFS stage (right) on the $x$-axis.

\section{DISCUSSION}

Endometriosis is defined as the presence of endometrial-like tissue outside the uterine cavity. It is a common, chronic, progressive and inflammatory disease, associated with local and systemic altered immune system ${ }^{(7)}$. The areas of endometriosis bleed each month, resulting in inflammation and scarring ${ }^{(8)}$.

The first important step in the pathophysiology of endometriosis is activation of the innate immune system. Increased release of inflammatory cytokines is a major part of the immunologic response. It has been demonstrated that some cytokine expression, including interleukins IL1, IL-2, IL-4, IL-5, IL-6, IL-8, IL-10, and IL-13, in the peritoneal and follicular fluid of women with endometriosis differs from that of women without this disease ${ }^{(9)}$. Moreover, some cytokines, especially IL-8, appear to have the potential to work as biomarkers to identify patients with endometriosis ${ }^{\mathbf{( 1 0})}$.

Interleukin 37 (previously called IL-1F7) is a newly discovered member of the IL-1 family. Interleukin 37 is located both in the cytoplasm and the nucleus ${ }^{(11)}$. The key role of IL-37 is inhibiting both innate and adaptive immunity by decreasing expression of pro-inflammatory cytokines ${ }^{(4)}$.
Anti-inflammatory activity of IL-37 requires IL-18R $\alpha$ and IL-1R8 to function together ${ }^{(12)}$. The behaviour and pathophysiological role of IL-37 in endometriosis is still unclear, so this study aimed to investigate gene and protein expression levels of IL37 in eutopic and ectopic endometrium of women with endometriosis in comparison with controls.

46 women were included in the study. The process of recruitment and handling the study population during the course of the study was done according to inclusion and exclusion criteria. 19 women in the endometriosis group and 11 women in the control group were excluded throughout the course of the study due to inadequate samples, histologically out-of-phase (endometrium was not in its proliferative phase), endometrial hyperplasia, endometritis or loss during follow up (confirmation of endometriosis by histopathology). The recruited 46 women included in this study were 23 women with endometriosis planned for laparoscopy assessed for eligibility and 23 control women free of endometriosis planned for laparoscopy for any other gynecological cause assessed for eligibility.

Our study found that there was no statistically significant difference between women of endometriosis and the control group regarding age 
with mean of $26.95 \pm 5.98$ and $\mathrm{p}$ value of 0.87 . Moreover our study found that there was no statistically significant difference between women of endometriosis group and the control group regarding parity with median (IQR) of 0-1. Fan et al. ${ }^{(14)}$ are in agreement with our study where their study found no statistically significant difference between women of endometriosis group and control group regarding parity with median of $0.9 \pm 0.8$ in stage 1 and 2, 0.6 \pm 0.5 in stage 3 and 4 and $0.9 \pm 0.6$ in control group with $\mathrm{p}$ value $>0.05$. In agreement with our study, Kaabachi et al. ${ }^{(13)}$ compared peritoneal fluid and blood samples from 30 women with endometriosis to those obtained from 20 women without endometriosis (control group). They found no statistically significant differences between both groups regarding parity as all were with unexplained infertility.

Our study found that there was no statistically significant differences regarding indications for laparoscopy between neither groups ( $\mathrm{P}$ value 0.55 ) nor regarding r-AFS score in endometriosis group. The range was from 4 to 56; and Median (IQR) was 14 (9.5 - 24.5). In endometriosis group $\mathrm{r}$-AFS staging was $4(18.1 \%)$ in stage I, $8(36.3 \%)$ in stage II, $8(36.3 \%)$ in stage III and $3(9.0 \%)$ in stage IV with noted considerable overlap in the performed procedures in the participants, e.g. in some women, both ovarian cystectomy and tubal disconnection were done in the same setting. Fan et al. ${ }^{(14)}$ are in agreement with our study regarding indications for laparoscopy. Their study found no statistically significant differences regarding indications for laparoscopy between both groups ( $\mathrm{P}$ value $>0.05$ ). In addition, the study group was in different stages of endometriosis, control group was free of endometriosis, and diagnosis was confirmed after laparoscopy by histopathology. Besides, Kaabachi $\boldsymbol{e t}$ al. ${ }^{(13)}$ are in agreement with us where their study found no statistically significant differences regarding indications for laparoscopy. The study compared peritoneal fluid and blood samples from 30 women with endometriosis to those obtained from 20 women without endometriosis acting as control that were confirmed after laparoscopy by histopathology. Both groups underwent laparoscopy for unexplained infertility. Additionally, in agreement with our study, Jiang et $\boldsymbol{a l} .{ }^{\left({ }^{(6)}\right.}$ in their study found no statistically significant difference regarding indications for laparoscopy through studying ectopic endometrial lesions obtained from 24 women with ovarian endometriosis underwent laparoscopic treatment for infertility and/or ovarian cysts.

Our study found that there was statistically significant difference regarding normalized target gene expression levels of IL-37 in eutopic endometrial tissues between the endometriosis and control groups with ranges of $8.50-45.60$ and $0.33-$ 11.38 respectively, mean of $20.78 \pm 10.32$ and $5.40 \pm$
2.94 respectively and $95 \%$ confidence interval 16.21 25.36 and $4.12-6.67$ respectively ( $\mathrm{P}$ value $<0.001$ ). In agreement with our study, Jiang et al. ${ }^{(6)}$ showed that messenger RNA (mRNA) levels of patients with ovarian endometriosis were statistically significantly higher than in control $(\mathrm{p}=0.005)$. In addition, Jiang $\boldsymbol{e t}$ $\boldsymbol{a l}^{\left({ }^{(15)}\right.}$ studied the levels of interleukins (IL)-1 $\beta$, IL-6, IL-10, and IL-37 in the sera of women with and without endometriosis and found that the levels of IL$1 \beta$, IL-6, IL-10, and IL-37 were higher in the endometriosis group than in the control group, and they were correlated with the stage of endometriosis. The area under the curve (AUC) for the IL-37 was 0.897 for the serum, while the AUC for the IL- 6 was 0.905 for the serum these results suggested that the serum IL-6 and IL-37 levels were significantly increased in the endometriosis patients, indicating that these cytokines may serve as biomarkers for the diagnosis of endometriosis. Moreover in agreement with our study, Fan et al. ${ }^{(14)}$ found significantly higher levels of serum IL-37 and IL-10 detected in patients with endometriosis group compared to control group $(\mathrm{p}<0.01)$. The sensitivity and specificity of serum IL-37 for distinguishing endometriosis were $81.48 \%$ and $83.33 \%$, respectively, and the cut off concentration was $69.84 \mathrm{pg} / \mathrm{ml}(\mathrm{p}<$ 0.01) (AUC $1 / 4$ 0.8930). Though Fan et al. (14) investigated expression of IL-37 in serum obtained from patients with endometriosis, while our study investigated it in eutopic endometrium in patient group compared to control group.

Our study found that there was statistically significant differences regarding normalized target gene expression levels in ectopic endometrium of the endometriosis compared to eutopic endometrium. In agreement with our study, Jiang et al. ${ }^{(6)}$ who studied IL-37 in the ectopic and eutopic endometrium in women with ovarian endometriosis where lesions were obtained from 24 women underwent laparoscopy.

\section{CONCLUSION}

This study aimed to investigate gene and protein expression levels of IL-37 in eutopic and ectopic endometrium of patients with endometriosis in comparison with control. The study found statistically significant difference regarding normalized target gene expression levels in both eutopic and ectopic endometrial tissues.

\section{REFERENCES}

1. Giudice L, Burney R, Becker C et al. (2019): Genetics and Genomics of Endometriosis. In Human Reproductive and Prenatal Genetics, Pp: 399-426. https://www.researchgate.net/publication/330048041_Ge netics_and_Genomics_of_Endometriosis

2. Bouaziz J, Mashiach R, Cohen S et al. (2018): How Artificial Intelligence Can Improve Our Understanding of the Genes Associated with Endometriosis: Natural 
Language Processing of the PubMed Database. Biomed Res Int., 2018: 6217812.

3. Kubatova A, Erdem A, Erdem $M$ et al. (2013): Serum cytokine and growth factor levels in patients with endometriosis. Centr Eur J Immunol., 38: 500-504.

4. Bulau, GrAuschka A, Schwaiger R et al. (2014): AntiIL-37 antibody abrogates the protection in IL-37 transgenic mice to LPS-induced septic shock. Cytokine, 63 (3): 250-6.

5. Dinarello C, Nold-Petry C, Nold M et al. (2016): Suppression of innate inflammation and immunity by interleukin-37. Eur. J. Immunol., 46: 1067-1081.

6. Jiang JF, Deng Y, Xue W et al. (2016): Increased Expression of Interleukin 37 in the Eutopic and Ectopic Endometrium of Patients with Ovarian Endometriosis. Reprod Sci., 23 (2): 244-8.

7. Wu J, Xie H, Yao S et al. (2017). Macrophage and nerve interaction in endometriosis. Journal of NeuroInflammation, 14 (1): 53-58.

8. Bulletti C, Coccia M, Battistoni S et al. (2010). Endometriosis and infertility. J Assist Reprod Genet., 27 (8): 441-7.

9. Eisenberg V, Zolti M, Soriano D (2012): Is there an association between autoimmunity and endometriosis? Autoimmun Rev., 11: 806-814.
10. Kalu E, Sumar N, Giannopoulos T et al. (2007): Cytokine profiles in serum and peritoneal fluid from infertile women with and without endometriosis. The Journal of Obstetrics and Gynaecology Research, 33: 490-5.

11. Nold M, Nold-Petry C, Zepp J et al. (2010): IL-37 is a fundamental inhibitor of innate immunity. Nat Immunol., 11 (11): 1014-24.

12. Nold-Petry C, Lo C, Rudloff I et al. (2015): IL-37 requires the receptors IL-18R $\alpha$ and IL-1R8 (SIGIRR) to carry out its multifaceted anti-inflammatory program upon innate signal transduction. Nat Immunol., 16 (4): 354-65.

13. Kaabachi W, Kacem O, Belhaj R et al. (2017): Interleukin-37 in endometriosis. Immunol Lett., 185: 5255.

14.Fan Q, Cheng C, Hackett C et al. (2010): Akt and autophagy cooperate to promote survival of drugresistant glioma. Science Signaling, 3: 81-89.

15. Jiang J, Jiang Z, Xue M (2019): Serum and peritoneal fluid levels of interleukin-6 and interleukin-37 as biomarkers for endometriosis. Gynecol Endocrinol., 35 (7): 571-575. 